\title{
The crystal and the rose: On the impact of crystals and crystallography on arts and mind
}

\author{
J.M. García-Ruiz \\ Laboratorio de Estudios Cristalográficos. Instituto Andaluz de Ciencias de la Tierra, Consejo Superior de Investigaciones \\ Cientificas-Universidad de Granada, Av. De las Palmeras 4, 18100 Armilla (Granada) Spain.
}

Juanma.garciaruiz@gmail.com

The belief in the existence of a sharp boundary dividing the world of symmetry into the realm of biology and sensuality and the realm of minerals and cold rationality has had a crucial impact on studies on early life detection on Earth and elsewhere. I have explored the origin of this secular antinomy and found that such a fictitious boundary has permeated the landscape of arts and philosophy for centuries [1]. It is shown that crystals and crystallographic theories have played a crucial role in the intellectual construction of that presumed boundary. The antinomy is illustrated with a debate between the young poet Federico García-Lorca and the young artist Salvador Dali, an archetypal debate to which crystals and what they evocate were central. It is concluded that along with the invaluable contribution of crystallography to the advancement of science and technology of art preservation, the notion of crystal transcended scientific thinking to inspire the arts, from literature to painting, from architecture to dance, from music to filmmaking. Thus, the very idea of crystal and crystallographic theories has been highly influential in the world of arts, architecture, and culture. The importance and the consequences that the crystalline order has had in the conformation of the consciousness, in the conception of the world, and the history of arts goes beyond what has
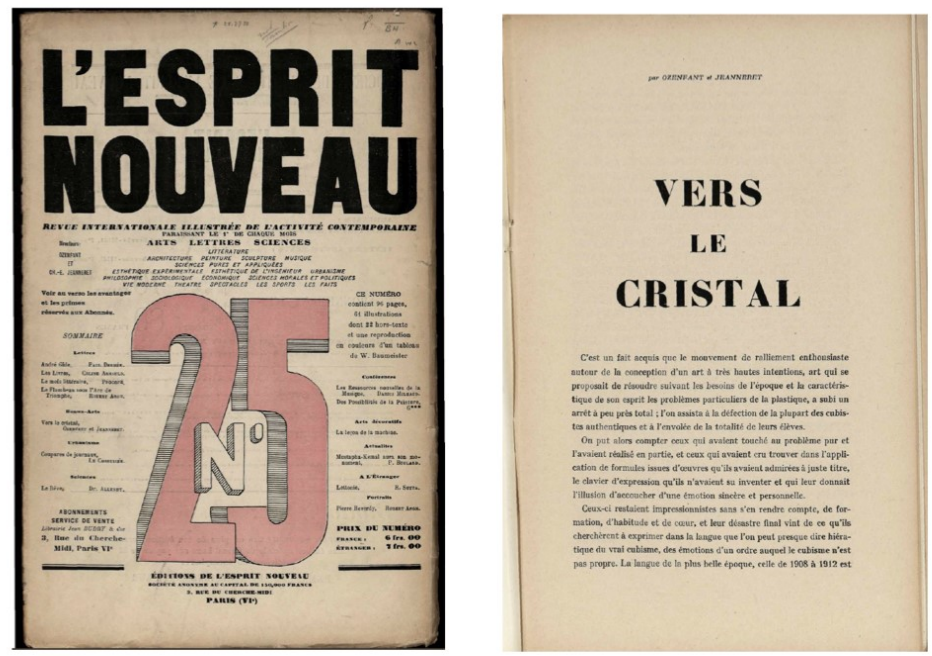

Figure 1. Cover of the issue 25 of L'Esprit Nouveau where A. Ozenfant and Le Corbusier published the article "Towards the crystals".

been considered a simple metaphor, and it is a subject that needs to be further.

This influence has evolved throughout history in correlation with increasing scientific knowledge about crystals. Since the origin of consciousness, hundreds of thousands of years ago, human fascination for crystals has been so deeply rooted in our brains as to shape our first symbolic behavior and perception of patterns. During prehistory, crystals had teleological and theological connotations derived from a hidden power of their singularity among the natural objects. Later on, from the classical world to the emergence of positive science in the eighteenth century, scholars and experts endorsed mineral crystals with healing powers. The sheer beauty of the external forms of crystals and everything it evokes fascinated illustrated people at that time. But the higher impact of crystals on the mind and cultures started in the 19 th century, when it was demonstrated the extraordinary link between the external harmony, redundantly beautiful symmetry of crystals, and the perfect internal order, periodic and iterative. Since then, the word crystal is full of evocations such as purity, transparency, beauty, equilibrium, rationality, intelligence, energy, and power [2].

[1] García-Ruiz, J. M. (2018). Substantia 2, 19.

[2] García-Ruiz, J.M. et al. (2021). IUCrJ. Submitted.

Keywords: History of crystallography; Primitive life detection; Crystal symbolism; Crystals and art

This investigation has received funding from the European Research Council under the European Union's Seventh Framework Programme (FP7/2007-2013)/ERC grant agreement $n^{\circ}$ 340863, and from the Ministry of Economy and Competitiveness of Spain (Program Salvador de Madariaga). 